\title{
HEAT DISSIPATION AND RIPPLE CURRENT RATING IN ELECTROLYTIC CAPACITORS
}

\author{
F. G. HAYATEE \\ College of Engineering, University of Mosul, Mosul, Iraq
}

(Received September 24, 1974)

\begin{abstract}
The ripple current rating in electrolytic capacitors is limited by the maximum allowable temperature rise inside the capacitor. The temperature rise is determined by the $I^{2} R$ losses inside the capacitor and the efficiency of heat flow from the interior to the surrounding. The ripple current rating can be extended by either reducing the $\tan \delta$ of the capacitor or by increasing the efficiency of heat flow to ambient.

The heat flow is determined by the thermal characteristics of the capacitor surface and thermal conductivity of the medium separating the capacitor winding from the surrounding.

In this article a mathematical analysis for the heat flow in capacitors is given. The effects of various parameters are examined and methods of extending the ripple current rating are discussed.
\end{abstract}

\section{INTRODUCTION}

The ripple current is a parameter which is of interest to both designers and users of electrolytic capacitors. This is especially true for large electrolytic capacitors used for smoothing in computer power supplies.

The maximum permissible ripple current in a capacitor is limited by various factors, such as the capacity of leads, lugs and terminals to withstand the rated current. However, the most important limitation is the maximum allowable temperature rise inside the capacitor. The temperature limit is imposed to prevent rapid deterioration in the performance of the capacitor resulting from increased electrochemical reaction between electrolyte and foil, and also to prevent damage to the alumina, the dielectric material.

The ripple current rating is normally evaluated empirically. Nomographs relating the parameters of a capacitor and its case size to the ripple rating have been given by McManus (1969) and Everett (1971). The formula used by McManus can be described by the relationship:

$$
I=3.1 \times\left(\frac{A \Delta T}{R}\right)^{1 / 2}
$$

where $I$ is the ripple current in Amps

$A$ is the surface area of the case in $\mathrm{m}^{2}$

$R$ is the series resistance of capacitor in $\Omega$

$\Delta T$ is the temperature rise in ${ }^{\circ} \mathrm{C}$.

Mason (1970), on the other hand, has used the relationship

$$
I \cong 2 \times 10^{2} A \sqrt{ } \Delta T
$$

to describe the rating in long life capacitors with working voltage in the range $6.3-100 \mathrm{~V}$.

For capacitors with large area of case to volume ratio, Campbell (1971) has suggested the rating to be proportional to volume at the rate of $8 \times 10^{4} \mathrm{~A} / \mathrm{m}^{3}$.

The determination of ripple current rating is based in this paper on a theoretical treatment of the heat conduction process in capacitors. The experimental work has permitted determination of relevant thermal parameters of the capacitor, and definition of areas of improvement that should be tackled to achieve efficient heat flow to ambient.

\section{THEORETICAL}

Examination of temperature variations at various parts of the capacitor indicates that the temperature of the cartridge is almost constant $\left( \pm 1^{\circ} \mathrm{C}\right)$ across its diameter and along its length. This is also true for the casing. It is therefore reasonable to assume that cartridge and casing are isothermal bodies.

The capacitor can therefore be treated as two concentric isothermal cylinders at temperature $T_{1}$ and $T_{2}$. Furthermore. heat flow can be assumed to be radial. This is a reasonable assumption for large capacitors whose cylindrical surface area is much greater than the end areas.

Under steady state conditions, radial heat flow can 


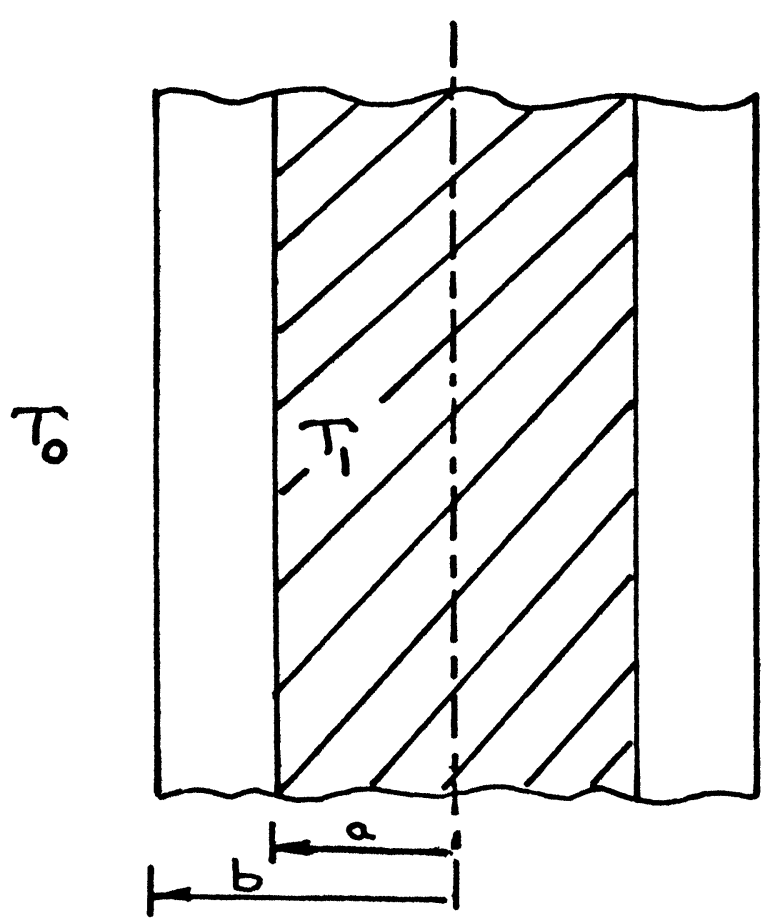

FIGURE 1 Two concentric cylinders of radii a and b with the inner cylinder at temperature $T_{1}$ and the ambient at temperature $T_{0}$.

be described by the differential equation:

$$
\frac{1}{r} \frac{\partial}{\partial r}\left(r \frac{\partial T}{\partial r}\right)=0
$$

which has the general solution

$$
T=A+B \ln r
$$

\subsection{Heat Loss From a Bare Can}

The constants $A$ and $B$ in Eq. (2) can be evaluated by applying appropriate boundary conditions. These are:

$$
T=T_{1} \quad \text { at } \quad r=a
$$

and

$$
\frac{\mathrm{d} T}{\mathrm{~d} r}=-\frac{G_{0}}{K_{0}}\left(T-T_{0}\right) \quad \text { at } \quad r=b
$$

where $T_{0}$ is the ambient temperature

$G_{0}$ is the exterior conductivity of the outer surface of a bare can

$K_{0}$ is the thermal conductivity of the medium separating the two cylinders.

The second boundary condition is true for radiation and forced convection, i.e. Newton cooling. Sub- stituting in (2) the complete solution becomes

$$
T_{2}=T_{0}+\frac{T_{1}-T_{0}}{1+\frac{G_{0}}{K_{0}} b \ln \left(\frac{b}{a}\right)}
$$

If the rate of internal heat generation is $Q_{0}$, it can be shown that

and

$$
T_{1}=T_{0}+\frac{Q_{0}}{2 \pi r_{0} b L}\left[1+\frac{G_{0}}{K_{0}} b \ln \left(\frac{b}{a}\right)\right]
$$

$$
T_{2}=T_{0}+\frac{Q_{0}}{2 \pi G_{0} L b}
$$

where $T_{2}$ is the temperature of the outer cylinder and $L$ is the length of the cylinders.

\subsection{Effect of Sleeving}

In practice, capacitors are normally sleeved with an insulating material such as PVC. The above analysis can be repeated for the sleeved can. Assuming that the rate of heat flow through the sleeving is $Q_{s}$ and the thickness of sleeving is $t(t \ll b)$ then

$$
T_{2 S}=T_{0}+\frac{Q_{s}}{2 \pi b L}\left(\frac{1}{G_{s}}+\frac{t}{K_{s}}\right)
$$

where $T_{2 S}$ is the temperature of the outer can when sleeved.

$G_{s}$ is the exterior conductivity of the sleeving and

$K_{s}$ is the thermal conductivity of the sleeving.

For the same amount of generated heat for bare and sleeved capacitor $\left(Q_{0}=Q_{s}\right)$ the temperature difference in the outer cylinder can be found using Eq. (5) and (6) and by eliminating To:

$$
T_{2}-T_{2 S}=\frac{Q_{0}}{2 \pi b L}\left[\frac{1}{G_{0}}-\left(\frac{1}{G_{s}}-\frac{t}{K_{s}}\right)\right]
$$

Equation (7) implies that the sleeving lowers the temperature and the outer can only if

$$
t<K_{s}\left(\frac{1}{G_{0}}-\frac{1}{G_{s}}\right)
$$

The temperature $T_{1 S}$ of the cartridge with sleeved case can be found in terms of the generated heat $Q_{s}$. The heat flow equation is:

$$
Q_{s}=\frac{2 \pi L K\left(T_{1 S}-T_{2 S}\right)}{\ln \left(\frac{b}{a}\right)}
$$


Eliminating $T_{2 S}$ from (6) and (9) we obtain

$$
T_{1 S}=T_{0}+\frac{Q_{s}}{2 \pi b L}\left[\frac{1}{G_{s}}+\frac{t}{K_{s}}+\frac{b}{K_{0}} \ln \left(\frac{b}{a}\right)\right]
$$

If the maximum temperature inside the capacitor is to be the same for the sleeved and the bare capacitor, the ratio of maximum permissible generated heat will be found from Eqs. (4) and (10). Thus,

$$
\frac{Q_{s}}{Q_{0}}=\frac{\frac{1}{G_{0}}+\frac{b}{K_{0}} \ln \left(\frac{b}{a}\right)}{\frac{1}{G_{s}}+\frac{t}{K_{s}}+\frac{b}{K_{0}} \ln \left(\frac{b}{a}\right)}
$$

\section{EXPERIMENTAL WORK}

Initial investigation on heat flow from cylindrical surfaces was carried out using the arrangement shown in Figure 2. Heat was generated inside the can using a

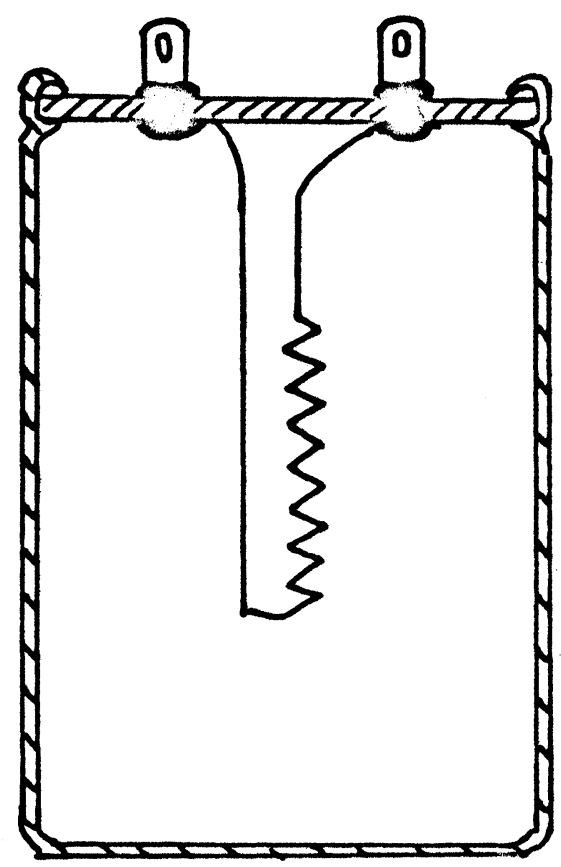

FIGURE 2 Heating element can assembly.

heating element rivetted to tag connections through the insulating top deck. An electronic thermometer (Comark type 1601) was used to measure the temperature.
A capacitor was simulated by wrapping an inner cylinder in three layers of craft tissue impregnated with electrolyte. This arrangement, resembling the cartridge of a capacitor, was placed inside an outer can. The two cans were separated at the base by several layers of impregnated tissue totalling $3 x$ $10^{-3} \mathrm{~m}$ in thickness.

The quantity of heat generated inside was varied, and the temperature was monitored on the inner and outer cylinders. The experiment was carried out for both bare and sleeved cans.

\section{RESULTS AND ANALYSIS}

\subsection{Determination of $G_{0}$ and $G_{s}$}

The variation of surface temperature on bare and sleeved cans as a function of $I^{2}$, and hence on generated internal heat was investigated. The two cans were of similar size, $3.5 \times 10^{-2} \mathrm{~m}$. in diameter and $8 \times 10^{-2} \mathrm{~m}$. long.

The dependence of $T_{2}$ and $T_{2 S}$ on $I^{2}$ is found to be linear up to approximately $50^{\circ} \mathrm{C}$ (Figure 3 ). This

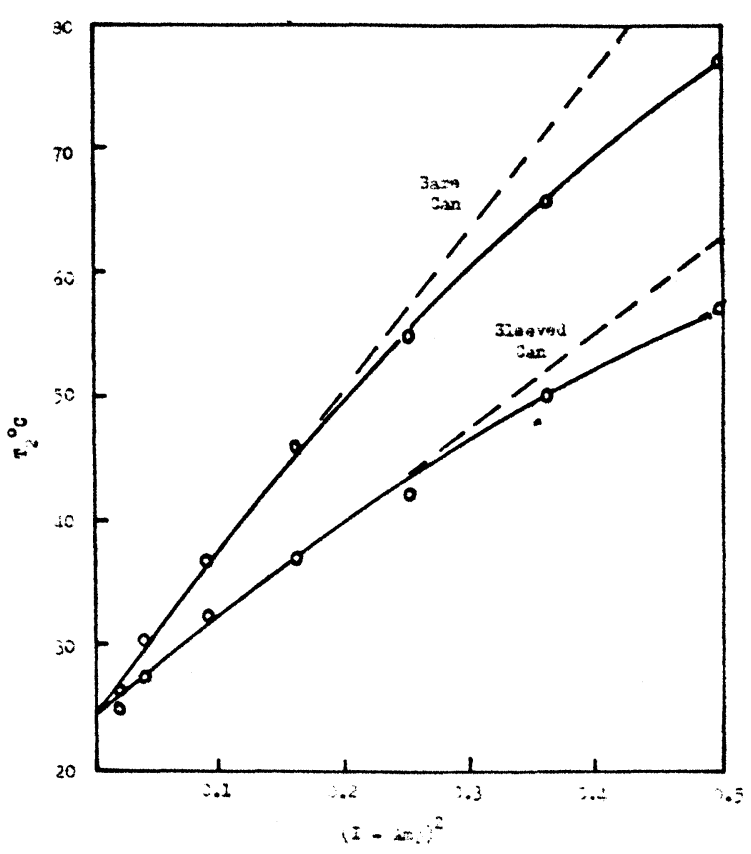

FIGURE 3 Dependence of the surface temperature $T_{2}$ of the sleeved and bare cans on the square of the current. 
dependence can be interpreted according to Eq. (5) and (6). The small deviation observed at higher temperatures is probably due to the assumption that the cooling is of the Newtonian type. In fact, the process is governed by the laws of natural cooling. The difference between the two processes at small temperature gradients is insignificant ${ }^{5}$.

The exterior conductivities of bare and sleeved cans can be found from Figure 3 using Eq. (5) and (6). The latter equation, however, contains $K_{s}$, the thermal conductivity of the sleeving. This was determined by measuring the temperature gradient across the sleeving for a given rate of heat flow. $K_{s}$ was found to be $1.0 \times 10^{-2} \mathrm{~W} / \mathrm{m} /{ }^{\circ} \mathrm{C}$. Subsequently $G_{0}$ and $G_{s}$ were evaluated from the slopes of curves in Figure 3. They were found to be:

$$
\begin{aligned}
& G_{0}=5.3 \mathrm{~W} / \mathrm{m}^{2} /{ }^{\circ} \mathrm{C} \\
& G_{s}=7.4 \mathrm{~W} / \mathrm{m}^{2} /{ }^{\circ} \mathrm{C} .
\end{aligned}
$$

\subsection{Heat Flow in Capacitors}

A capacitor was simulated by wrapping the bare can with electrolyte impregnated tissue and placing it in a larger can $\left(5 \times 10^{-2} \mathrm{~m}\right.$ diameter $\times 11 \times 10^{-2} \mathrm{~m}$ long). To ensure a minimum heat loss from the base the arrangement was placed on a thermal insulating material.

The temperature on the inner and outer cylinders was measured as a function of dissipated power for both bare and sleeved outer cans.

The dependence of $T_{2}$ on $I^{2}$ is shown in Figure 4.

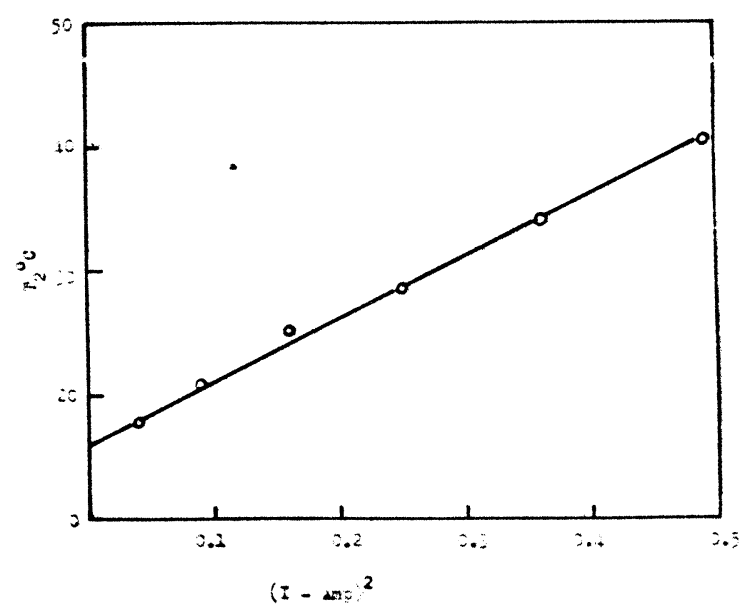

FIGURE 4 Dependence of the temperature $T_{2}$ of the bare outer can on the square of the current.
This is similar to the results shown in Figure 3 and it is in accordance with Eq. (5). The ratio of the slopes for the two curves of $T_{2}$ versus $I^{2}$ in Figures 3 and 4 is 2.4. Under identical test conditions this ratio should correspond to the ratio of surface area of the two cans. However, can dimensions yield a ratio of 2.1. The discrepancy is probably due to changes in the state of the ambient in the two tests, and small differences in the surface conditions of the two cans.

According to Eq. (3) there is a straight line relationship between $T_{1}$ and $T_{2}$. Using this to interpret the results presented in Figure 5 yields a value for $G_{0} / K_{0}$ of $110 \mathrm{~m}^{-1}$. Hence, the value of $K_{0}$ is found to be $4.8 \times 10^{-2} \mathrm{~W} / \mathrm{m} /{ }^{\circ} \mathrm{C}$. The dependence of the temperatures $T_{1 S}$ and $T_{2 S}$ of the inner and outer cans on internal heat generated when the outer can is sleeved is shown in Figure 6.

From these graphs the values of $G_{s}$ and $K_{0}$ are determined utilizing Eq. (6) and (10), and using the value of $K_{s}=4.6 \times 10^{-2} \mathrm{~W} / \mathrm{m} /{ }^{\circ} \mathrm{C}$ which has been determined previously. The analysis yields a value of $G_{s}=9.0 \mathrm{~W} / \mathrm{m} /{ }^{\circ} \mathrm{C}$ and $K_{0}=4.6 \times 10^{-2} \mathrm{~W} / \mathrm{m} /{ }^{\circ} \mathrm{C}$. This value of $G_{s}$ is $15 \%$ higher than the value obtained previously. It is possible that this difference is due to the difference in the thermal characteristics of the larger and small cans. $K_{0}$, on the other hand, is the property of the medium separating the inner and outer cans, and the new value is only $4 \%$ lower than that previously calculated.

The effect of the presence of bitumen, the material commonly used to hold the capacitor cartridge in the can, on the heat flow was investigated. The space between the inner and outer cans was half filled with bitumen. The results of Figure 7 shows hardly any effect of bitumen on the heat flow, except for a small change at high temperature gradients.

\section{RIPPLE RATING OF CAPACITORS}

Having determined the values of $K_{0}, G_{0}, K_{s}$ and $G_{s}$, it is possible to determine the amounts of dissipated power $Q_{0}$ or $Q_{s}$ that raise the temperature of the capacitor cartridge $T_{1}$ and $T_{1 S}$ above the ambient temperature $T_{0}$. If we are to allow $10^{\circ} \mathrm{C}$ temperature difference between the cartridge and the ambient, then according to Eq. (4):

Heat lost per unit area of can $=\frac{10 \times 5.3}{1+110 b \ln \left(\frac{b}{a}\right)} \mathrm{W} / \mathrm{m}^{2}$. 


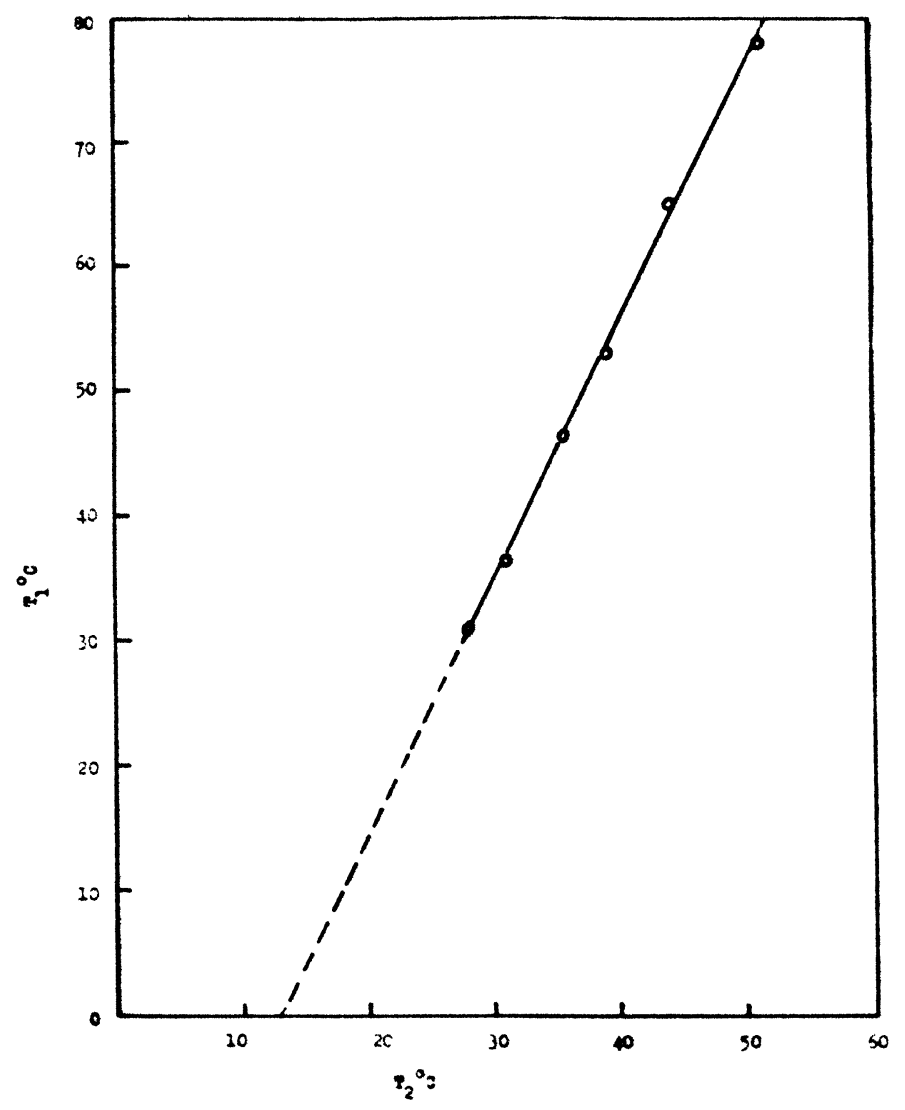

FIGURE 5 Relationship between the inner can temperature $T_{1}$ and outer can temperature $T_{2}$.

In practice, the separation between the can and the cartridge is usually made small, and thus the maximum heat lost is approximately $53 \mathrm{~W} / \mathrm{m}^{2}$. If, however, we consider a can with radius of $2 \times 10^{-2} \mathrm{~m}$ and a cartridge of radius $1.5 \times 10^{-2} \mathrm{~m}$, the heat flow will be $32 \mathrm{~W} / \mathrm{m}^{2}$, which is about $41 \%$ lower than the maximum value.

The sleeved can with PVC sleeving $1.5 \times 10^{-4} \mathrm{~m}$ thick, on the other hand, can dissipate according to Eq. (10) a maximum of $68 \mathrm{~W} / \mathrm{m}^{2}$. This is nearly $30 \%$ higher than the maximum dissipation of a bare can. It is therefore possible to increase the ripple current by sleeving by $15 \%$, provided that the separation of can and cartridge is small. This figure is a special case, and it applies to green PVC sleeving, $1.5 \times 10^{-4} \mathrm{~m}$ thick. The uprating factor is expected to change with the colour, surface texture and thickness of the sleeve. The power dissipated from a sleeved can of radius $2 \times 10^{-2} \mathrm{~m}$ enclosing a cartridge with radius $1.5 \mathrm{x}$ $10^{-2} \mathrm{~m}$ works out to be $39 \mathrm{~W} / \mathrm{m}^{2}$ and thus about $43 \%$ lower than the maximum obtainable value. In this case the ripple rating could be $10 \%$ higher than the corresponding bare assembly.

Most manufacturers limit the maximum internal temperature of the capacitor to $90^{\circ} \mathrm{C}$, and specify the ripple current rating at ambient temperature of $70^{\circ} \mathrm{C}$ or $85^{\circ} \mathrm{C}$. According to our analysis, the ripple rating is proportional to the square root of the temperature difference between interior of capacitor and the ambient. Thus, increasing the ambient from $70^{\circ} \mathrm{C}$ to $85^{\circ} \mathrm{C}$ should be accompanied by halving the ripple rating so as not to exceed the permissible internal temperature of $90^{\circ} \mathrm{C}$. Some manufacturers, in fact, do practice this rule. This implies, however, that the change in the equivalent series resistance (E.S.R.) of the capacitor with temperature is ignored. Fortunately, the rise in temperature lowers the resistance and therefore the rating at $85^{\circ} \mathrm{C}$ includes a safety margin. 


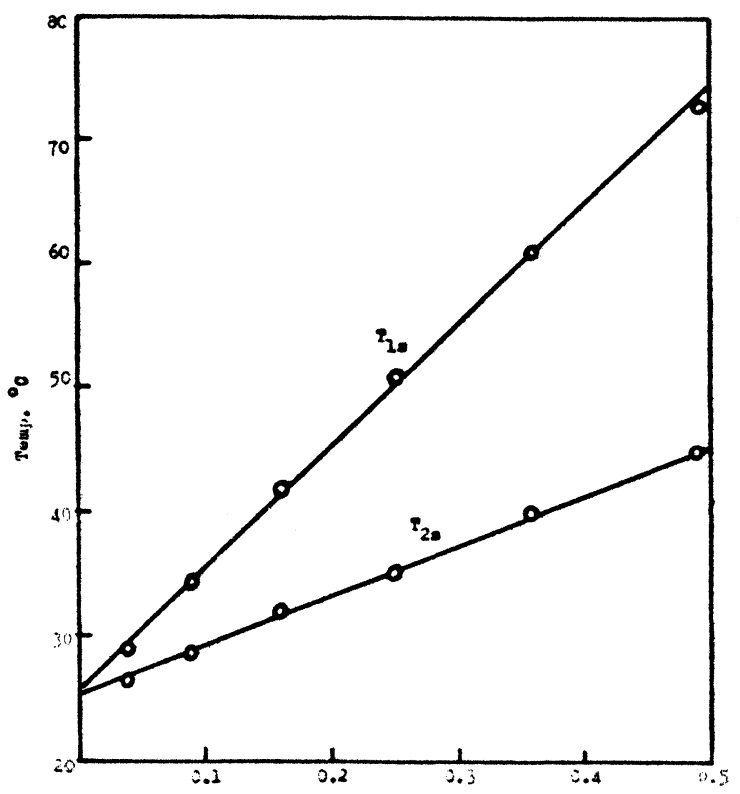

FIGURE 6 Dependence of temperature $T_{1} S$ of the inner can and $T_{2 \mathrm{~S}}$ of the sleeved outer can on the square of the current.

\section{CONCLUDING REMARKS}

The experimental results show consistency with the proposed simple model for heat flow in a capacitor. Sleeving of the capacitor leads to better heat dissipation to the ambient and thus it should be introduced in capacitors where ripple current is of importance. Importance must also be attached to the separation of the can from the cartridge. Minimizing the separation, or crimping the can on the cartridge can result in the uprating of the current by as much as $20 \%$.

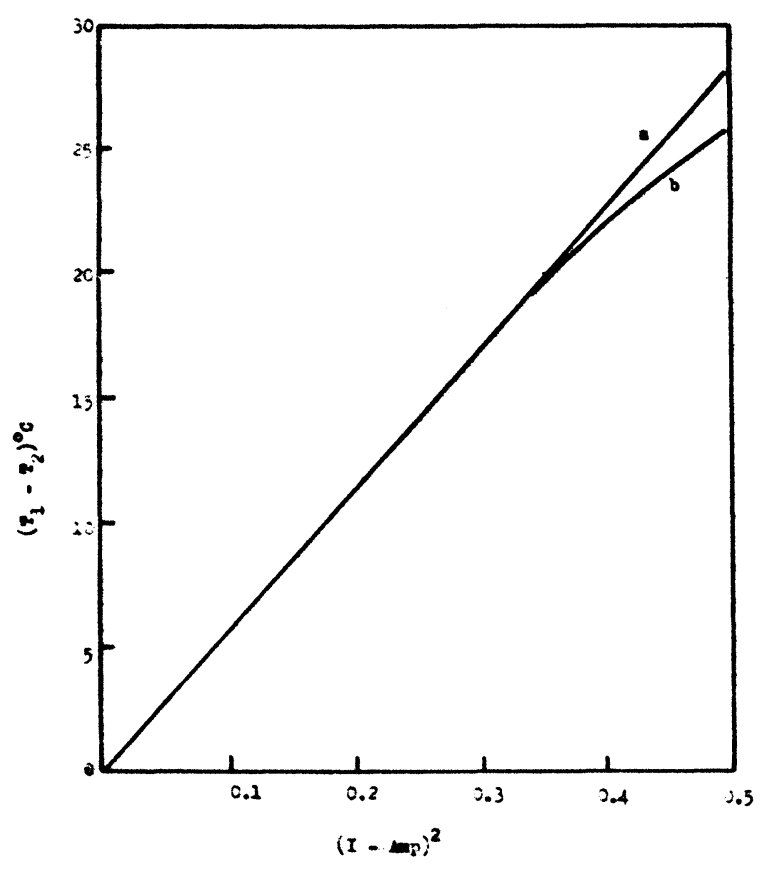

FIGURE 7 The temperature difference between inner and outer can as a function of the square of the current: $\begin{array}{ll}\text { (a) Without bitumen } & \text { (b) With bitumen. }\end{array}$

\section{REFERENCES}

1. R. P. McManus, Electron. Components, 2, 171 (1969).

2. R. J. Everitt, Electron. Equip. News, 2, 12 (1971).

3. D. Mason, Mullard Tech. Commun., 7, 122 (1970).

4. D. S. Campbell, Component Technol., 4, 8 (1971).

5. H.S. Carslaw, and J.C. Jaeger, Conduction of Heat in Solids, (Oxford University Press, London, 1947). 

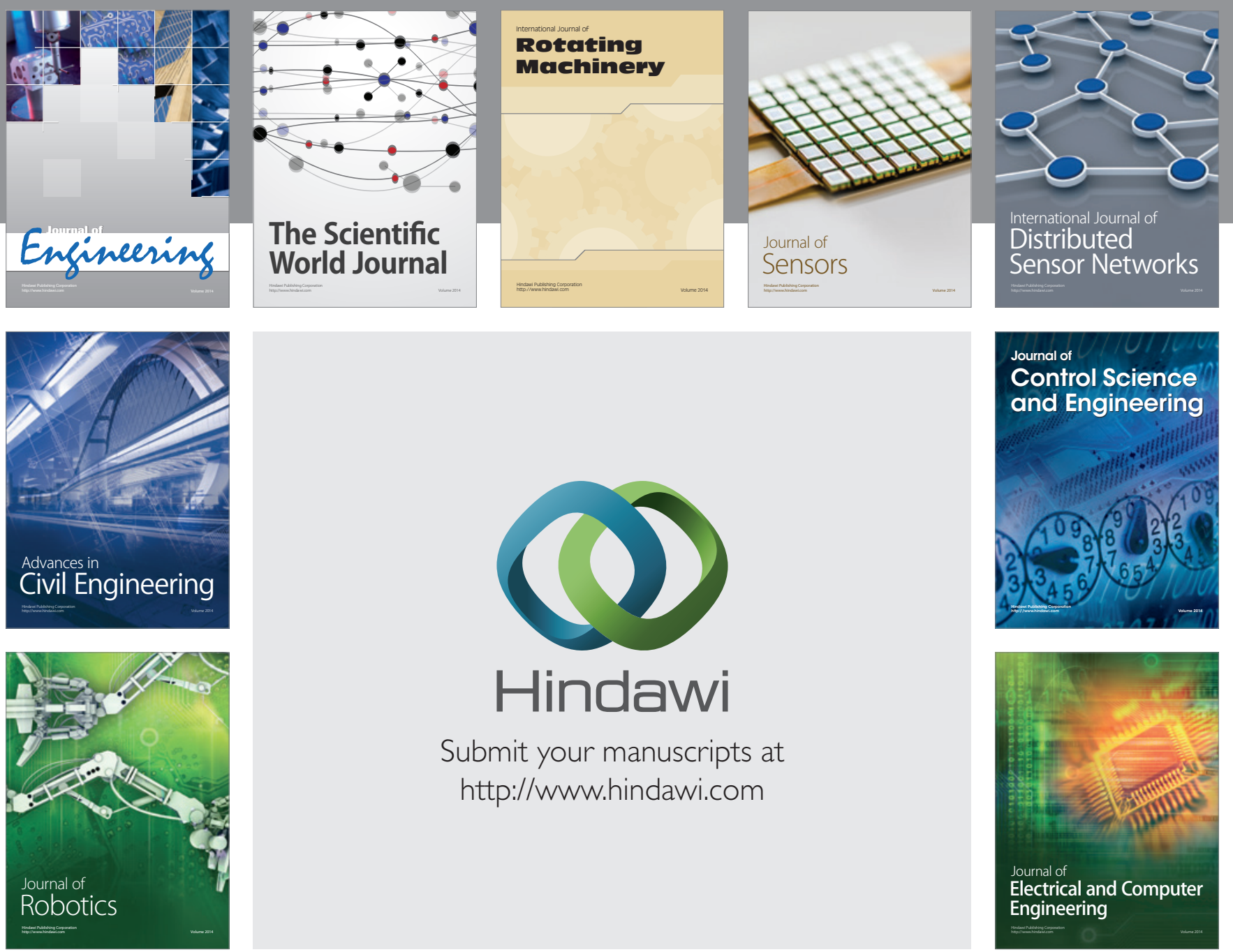

Submit your manuscripts at

http://www.hindawi.com
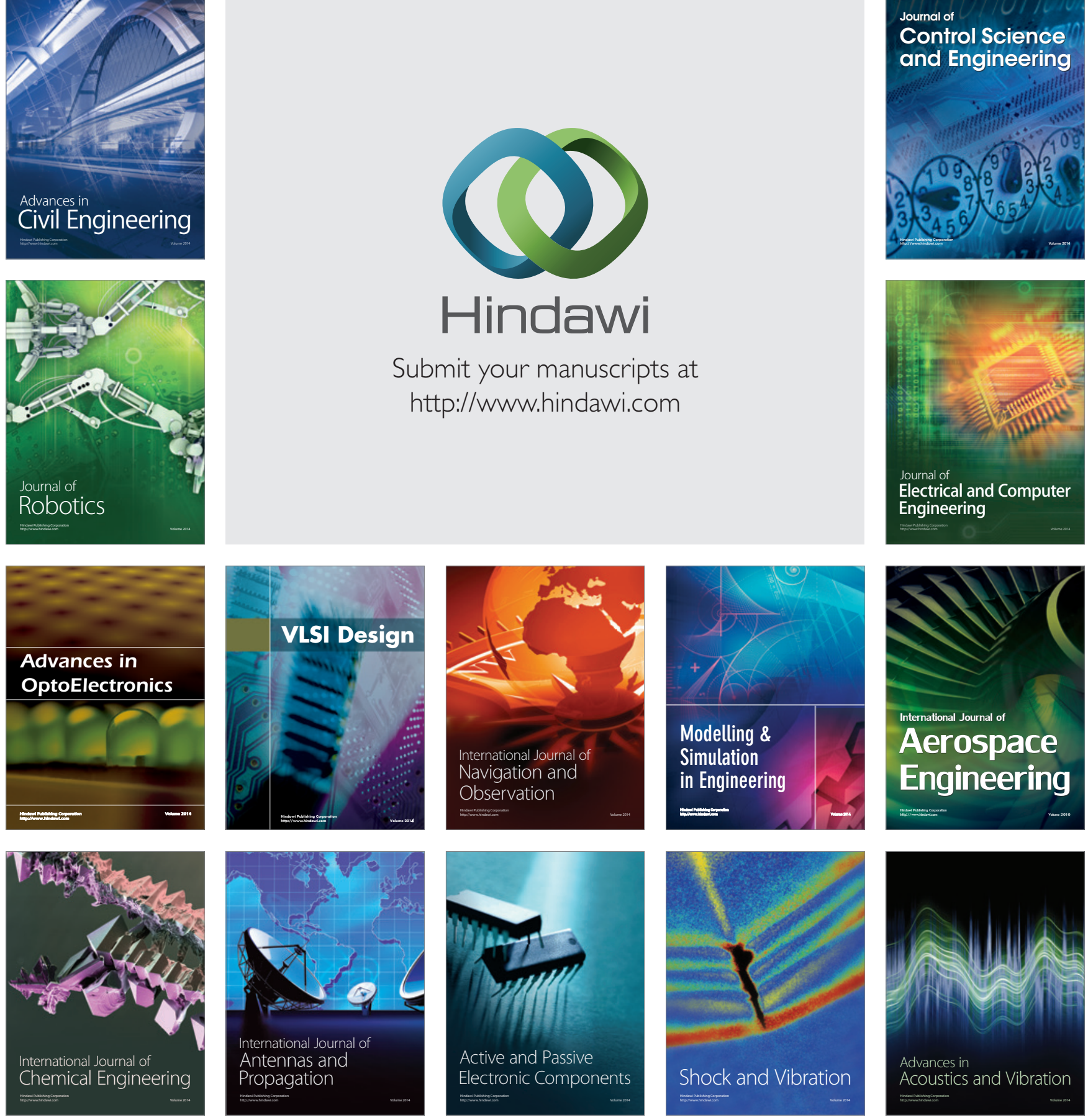